\title{
Coordinates Estimation of the Air Target in the Multiitem Observation System «Navigation Satellites - the Air Target - the Ground Receiver»
}

\author{
Vladislav V. Kiryushkin* and Denis A. Cherepanov \\ Military Education and Research Centre of Military-Air Forces \\ "Military-Air Academy \\ Named After Professor N.E. Zhukovsky and Yu.A. Gagarin» \\ 54a Starykh Bolshevikov Str., Voronezh, 394064, Russia
}

Received 23.06.2016, received in revised form 18.09.2016, accepted 17.10.2016

In present paper we offered the new way of coordinates estimation of the air target in the multiitem observation system "navigation satellites - the air target - the ground receiver». This way is based on the different-distance method of the navigation task solution in the receiver of Global navigating satellite system. The executed imitating modelling showed, that the accuracy of the air target site estimation, obtained by the ground receiver with use of the offered way, is less than 20 metres. This accuracy is commensurable with accuracy of an estimation of own coordinates of the receiver.

Keywords: multiitem observation system; Global navigating satellite system, different-distance measurements, method of the least squares.

Citation: Kiryushkin V.V., Cherepanov D.A. Coordinates estimation of the air target in the multiitem observation system «navigation satellites - the air target - the ground receiver», J. Sib. Fed. Univ. Eng. technol., 2016, 9(8), 1172-1182. DOI: $10.17516 / 1999-494 X-2016-9-8-1172-1182$.

(C) Siberian Federal University. All rights reserved

* Corresponding author E-mail address: kiryushkin.vlad@mail.ru 


\title{
Оценка координат воздушной цели
}

\author{
в многопозиционной системе наблюдения \\ «навигационные спутники - воздушная цель -
}

наземный приемник»

\author{
В.В. Кирюшкин, Д.А. Черепанов \\ Военный учебно-научный центр Военно-воздушных сил \\ «Военно-воздушная академия \\ имени профессора Н.Е. Жуковского и Ю.А. Гагарина» \\ Россия, 394064, Воронеж, ул. Старых Большевиков, 54 а
}

В статье предложен новый способ определения координат воздушной иели в многопозиционной неизлучающей системе наблюдения «навигационные спутники воздушная цель - наземный приемник», в основе которого лежит разностно-дальномерный метод решения навигационной задачи в аппаратуре потребителя глобальной навигационной спутниковой системы. Проведенное имитационное моделирование показало, что точность оценки местоположения воздушной иели, получаемой в наземном приемнике с использованием предложенного способа, соизмерима с точностью оченки собственных координат приемника и не превышает 20 м.

Ключевые слова: многопозииионная система наблюдения, глобальная навигачионная спутниковая система, разностно-дальномерные измерения, метод наименьших квадратов.

\section{Введение}

В современных условиях все большее распространение приобретают многопозиционные неизлучающие системы наблюдения с подсветом внешним электромагнитным полем. Это обусловлено, во-первых, значительно более высокой живучестью таких систем в сравнении с моностатическими радиолокационными системами, вероятность уничтожения которых противорадиолокационными средствами поражения очень высока, и, во-вторых, более высокой эффективностью многопозиционных систем при обнаружении малозаметных целей, построенных по технологии «стелс» [1]. При этом в качестве подсвета могут быть использованы сигналы различных радиотехнических систем: широковещательных радиостанций, станций ретрансляции телевизионных сигналов, базовых станций сотовых систем связи, а также навигационных спутников глобальной навигационной спутниковой системы (ГНСС) [2-7].

Наиболее перспективными представляются системы с подсветом навигационными сигналами ГНСС, так как в этом случае уже решены многие вопросы, связанные с синхронизацией и координатной привязкой передающих и приемных позиций системы [6-8].

В [8] была исследована энергетика навигационных сигналов, рассеянных воздушной целью, попавшей в зону действия бистатической системы «навигационный спутник - воздушная цель - наземный приемник», и обоснована возможность приема рассеянных сигналов в современном навигационном приемнике наряду с навигационными сигналами прямого распространения. В [9] предложен способ обнаружения рассеянного сигнала на основе алгоритма 
компенсационного приема полезного сигнала на фоне структурно-детерминированной помехи, а также устройство, его реализующее, как вариант модернизации современного навигационного приемника.

В настоящей статье предложен способ определения координат воздушной цели, попавшей в зону действия многопозиционной системы наблюдения «навигационные спутники - воздушная цель - наземный приемник», как основа построения канала вторичной обработки модернизированного навигационного приемника системы наблюдения.

\section{Постановка задач}

В многопозиционной неизлучающей системе наблюдения «навигационные спутники воздушная цель - наземный приемник» для подсвета воздушных целей используются сигналы навигационных спутников ГНСС $\mathrm{C}_{j}(j=1,2, \ldots, N$, где $N \geq 4)$ с координатами $\left(x_{j}, y_{j}, z_{j}\right)$ (рис. 1).

В наземном приемнике П с известными координатами $\left(x_{\Pi}, y_{\Pi}, z_{\Pi}\right)$ наряду с навигационными сигналами, распространяющимися вдоль прямого пути «j-й навигационный спутник - наземный приемник» с геометрической дальностью $r_{j}^{\Pi}$ (сигналами прямого распространения), принимаются навигационные сигналы, рассеянные воздушной целью Ц с неизвестными координатами $(x, y, z)$, находящейся в зоне действия многопозиционной системы наблюдения. Рассеянные навигационные сигналы выделяют на фоне навигационных сигналов прямого распространения одним из оценочно-корреляционно-компенсационных методов [9, 11]. По рассеянным сигналам осуществляется измерение дальностей $r_{j}^{\mathrm{P}}$ вдоль пути распространения «j-й навигационный спутник - воздушная цель - наземный приемник», соответствующее выражение для которых имеет следующий вид:

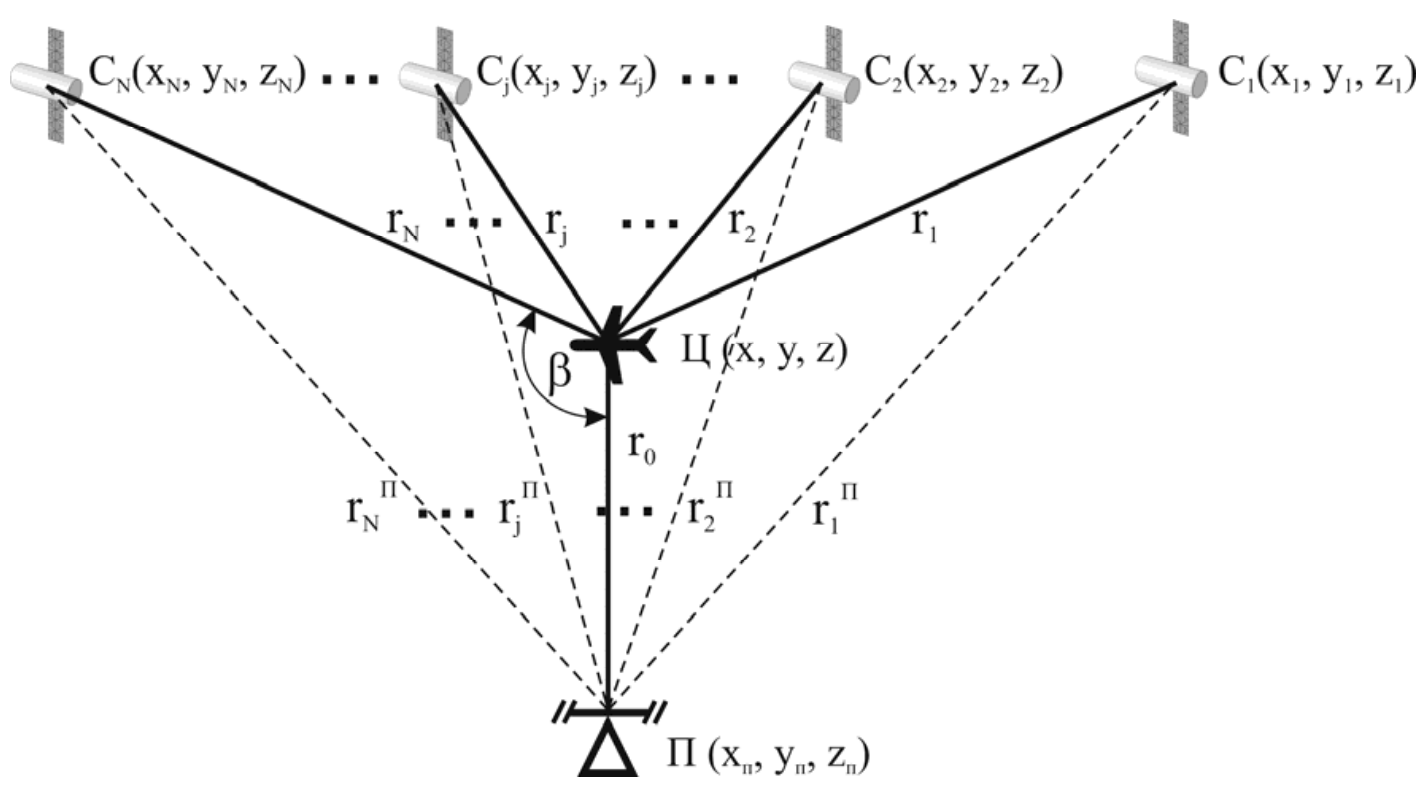

Рис. 1. Многопозиционная система наблюдения «навигационные спутники - воздушная цель - наземный приемник» 


$$
\begin{aligned}
& r_{j}^{\mathrm{P}}=r_{j}+r_{0}+\delta_{\text {п }}+\varepsilon= \\
& =\left[\left(x_{j}-x\right)^{2}+\left(y_{j}-y\right)^{2}+\left(z_{j}-z\right)^{2}\right]^{1 / 2}+ \\
& +\left[\left(x-x_{\text {п }}\right)^{2}+\left(y-y_{\text {п }}\right)^{2}+\left(z-z_{\text {п }}\right)^{2}\right]^{1 / 2}+\delta_{\text {п }}+\varepsilon,
\end{aligned}
$$

где $r_{j}$ - дальность пути «j-й навигационный спутник - воздушная цель»; $r_{0}$ - дальность пути «воздушная цель - наземный приемник»; $\delta_{\text {п }}$ - систематическая погрешность измерения дальности, обусловленная смещением шкалы времени приемника относительно бортовой шкалы времени спутников ГНСС; $\varepsilon$ - случайная погрешность измерения дальности.

На основании полученных измерений дальностей $r_{j}^{\mathrm{P}}$ требуется определить неизвестные координаты $(x, y, z)$ воздушной цели.

\section{Способ определения координат воздушной цели в многопозиционной системе наблюдения}

Предложенный способ определения координат воздушной цели, попавшей в зону действия многопозиционной системы наблюдения «навигационные спутники - воздушная цель - наземный приемник», основан на разностно-дальномерном навигационном методе определения координат в аппаратуре потребителя ГНСС [10].

Для реализации этого метода одно из полученных измерений (1), например измерение для 1-го спутника (выбрано условно и не устраняет общности), выбирается в качестве опорного и при составлении системы уравнений соответствующее выражение

$$
\begin{aligned}
& r_{1}^{\mathrm{P}}=r_{1}+r_{0}+\delta_{\text {п }}+\varepsilon= \\
& =\left[\left(x_{1}-x\right)^{2}+\left(y_{1}-y\right)^{2}+\left(z_{1}-z\right)^{2}\right]^{1 / 2}+ \\
& +\left[\left(x-x_{\text {п }}\right)^{2}+\left(y-y_{\text {п }}\right)^{2}+\left(z-z_{\text {п }}\right)^{2}\right]^{1 / 2}+\delta_{\text {п }}+\varepsilon
\end{aligned}
$$

вычитается из выражений для измерений всех остальных спутников.

В результате в левой части уравнений записываются разности измеренных дальностей

$$
\Delta r_{j 1}^{P}=r_{j}^{\mathrm{P}}-r_{1}^{\mathrm{P}},
$$

а в правой части уравнений после сокращений одинаковых по величине и противоположных по знаку слагаемых (за исключением случайных погрешностей измерений) остаются выражения для разностей расстояний, пройденных навигационными сигналами вдоль путей распространения «j-й навигационный спутник - воздушная цель» и «1-й навигационный спутник - воздушная цель», включающие известные координаты спутников и неизвестные координаты воздушной цели

$$
\begin{gathered}
{\left[\left(x_{j}-x\right)^{2}+\left(y_{j}-y\right)^{2}+\left(z_{j}-z\right)^{2}\right]^{1 / 2}+\left[\left(x-x_{\mathrm{n}}\right)^{2}+\left(y-y_{\mathrm{n}}\right)^{2}+\left(z-z_{\mathrm{n}}\right)^{2}\right]^{1 / 2}+\delta_{\text {п }}+\varepsilon-} \\
-\left[\left(x_{1}-x\right)^{2}+\left(y_{1}-y\right)^{2}+\left(z_{1}-z\right)^{2}\right]^{1 / 2}-\left[\left(x-x_{\mathrm{n}}\right)^{2}+\left(y-y_{\mathrm{n}}\right)^{2}+\left(z-z_{\mathrm{n}}\right)^{2}\right]^{1 / 2}-\delta_{\text {п }}-\varepsilon= \\
=\left[\left(x_{j}-x\right)^{2}+\left(y_{j}-y\right)^{2}+\left(z_{j}-z\right)^{2}\right]^{1 / 2}-\left[\left(x_{1}-x\right)^{2}+\left(y_{1}-y\right)^{2}+\left(z_{1}-z\right)^{2}\right]^{1 / 2}+\varepsilon . \\
-1175-
\end{gathered}
$$


Таким образом, для $N$ спутников, для которых выполняется условие просветной радиолокации (значение бистатического угла лежит в пределах $\beta=130-180^{\circ}$ [1], см. рис. 1), получают систему из $N-1$ уравнений

$$
\begin{aligned}
& \Delta r_{j 1}^{P}=\left[\left(x_{j}-x\right)^{2}+\left(y_{j}-y\right)^{2}+\left(z_{j}-z\right)^{2}\right]^{1 / 2}- \\
& -\left[\left(x_{1}-x\right)^{2}+\left(y_{1}-y\right)^{2}+\left(z_{1}-z\right)^{2}\right]^{1 / 2}+\varepsilon,
\end{aligned}
$$

где $j=2,3, \ldots, N$ - номер спутника.

Для решения полученной системы нелинейных уравнений воспользуемся итерационным методом наименьших квадратов, для чего запишем систему нелинейных уравнений (5) в обобщенном виде:

$$
\mathbf{R}=\mathbf{R}(\mathbf{q}, \mathbf{Q})
$$

где $\mathbf{R}-(N-1)$-мерный вектор измерений разностей дальностей; $\mathbf{q}=\left[\begin{array}{ll}x y z & z\end{array}\right]^{T}-$ вектор истинных координат воздушной цели; $\mathbf{Q}$ - матрица координат навигационных спутников.

Решение системы уравнений (6) методом наименьших квадратов представляет собой процесс многократной (итерационной) обработки результатов навигационных измерений по формуле

$$
\hat{\mathbf{q}}_{k}=\hat{\mathbf{q}}_{k-1}+\left(\mathbf{C}_{k-1}^{T} \mathbf{P} \mathbf{C}_{k-1}\right)^{-1} \mathbf{C}_{k-1}^{T} \mathbf{P} \delta \mathbf{R}_{k-1},
$$

где $\hat{\mathbf{q}}_{k-1}=\left(\hat{x}_{k-1}, \hat{y}_{k-1}, \hat{z}_{k-1}\right)^{T}$ - оценка вектора координат цели на $k-1$ итерации вычислений; $\delta \mathbf{R}_{k-1}=\mathbf{R}-\hat{\mathbf{R}}_{k-1}-$ вектор разностей (невязок), измеренных $\mathbf{R}$ и рассчитанных на $k-1$ итерации $\hat{\mathbf{R}}_{k-1}$ разностей дальностей; $\mathbf{C}_{k-1}$ - матрица частных производных от измеряемых навигационных функций по определяемым координатам; $k$ - номер итерационного цикла (как правило, не более 4), $\mathbf{P}$ - матрица, обратная корреляционной матрице погрешностей измерений дальностей [10].

Для бистатических разностно-дальномерных измерений элементы вектора невязок $\delta \mathbf{R}_{k-1}$ будут вычисляться по формулам (на примере измерений для $j$-го спутника):

$$
\begin{aligned}
& \delta\left(\Delta r_{j 1, k-1}^{P}\right)=\Delta r_{j 1}^{P}-\hat{r}_{j, k-1}+\hat{r}_{1, k-1}, \\
& \hat{r}_{j, k-1}=\left[\left(x_{j}-\hat{x}_{k-1}\right)^{2}+\left(y_{j}-\hat{y}_{k-1}\right)^{2}+\left(z_{j}-\hat{z}_{k-1}\right)^{2}\right]^{1 / 2}, \\
& \hat{r}_{1, k-1}=\left[\left(x_{1}-\hat{x}_{k-1}\right)^{2}+\left(y_{1}-\hat{y}_{k-1}\right)^{2}+\left(z_{1}-\hat{z}_{k-1}\right)^{2}\right]^{1 / 2} .
\end{aligned}
$$

При этом матрица наблюдений $\mathbf{C}_{k-1}=\left[\begin{array}{llll}\mathbf{C}_{21, k-1} & \ldots \mathbf{C}_{j 1, k-1} & \ldots \mathbf{C}_{N 1, k-1}\end{array}\right]^{T}$ вычисляется так же, как и для обычного разностно-дальномерного метода [10]:

$$
\mathbf{C}_{j 1, k-1}=\left[\begin{array}{lll}
\left(\cos \alpha_{j}-\cos \alpha_{1}\right) & \left(\cos \beta_{j}-\cos \beta_{1}\right) & \left(\cos \gamma_{j}-\cos \gamma_{1}\right)
\end{array}\right],
$$

где

$$
\begin{aligned}
\cos \alpha_{j}=\frac{x_{j}-\hat{x}_{k-1}}{\hat{r}_{j, k-1}} ; \cos \beta_{j} & =\frac{y_{j}-\hat{y}_{k-1}}{\hat{r}_{j, k-1}} ; \cos \gamma_{j}=\frac{z_{j}-\hat{z}_{k-1}}{\hat{r}_{j, k-1}}, \\
- & 1176-
\end{aligned}
$$




$$
\cos \alpha_{1}=\frac{x_{1}-\hat{x}_{k-1}}{\hat{r}_{1, k-1}} ; \cos \beta_{1}=\frac{y_{1}-\hat{y}_{k-1}}{\hat{r}_{1, k-1}} ; \cos \gamma_{1}=\frac{z_{1}-\hat{z}_{k-1}}{\hat{r}_{1, k-1}}
$$

Точность оценки вектора $\hat{\mathbf{q}}$ координат воздушной цели в наземном навигационном приемнике определяется корреляционной матрицей погрешностей [10]

$$
\mathbf{K}_{q}=\left(\mathbf{C}_{k-1}^{T} \mathbf{P C}_{k-1}\right)^{-1}
$$

\section{Методика исследования точности оценки координат воздушной цели в многопозиционной системе наблюдения}

Исследование точности оценки координат воздушной цели в многопозиционной системе наблюдения «навигационные спутники - воздушная цель - наземный приемник» с использованием предложенного разностно-дальномерного способа было проведено методом имитационного моделирования. Структурная схема имитационной модели приведена на рис. 2.

В состав имитационной модели входят: модель движения воздушной цели, модель движения навигационных спутников, формирователь вектора измерения, алгоритм определения вектора координат воздушной цели и блок оценки точности определения координат.

В качестве модели движения воздушной цели использовалась траектория полета самолета, сформированная в среде авиасимулятора FlightGear в виде массива значений вектора q истинных координат воздушной цели.

В основу модели движения навигационных спутников положена модель невозмущённого движения спутников [12], исходными данными для которой являются начальные значения орбитальных элементов, а на выходе модели в каждый момент времени формируется матрица $\mathbf{Q}$ координат навигационных спутников.

Формирователь вектора измерения реализует алгоритм определения «радиовидимых» относительно наземного приемника навигационных спутников ГНСС и формирует $(N-1)$-мерный вектор $\mathbf{R}$ измерений разностей дальностей в соответствии с (1) - (6) для навигационных спутников, расположение которых относительно наземного приемника и воздушной цели удовлетворяет условию просветной локации [1]. При этом считается, что случайные составляющие погрешности измерения дальности $\varepsilon$ представляют собой гауссовские шумы с нулевым математическим ожиданием и дисперсией, соответствующей точностным характери-

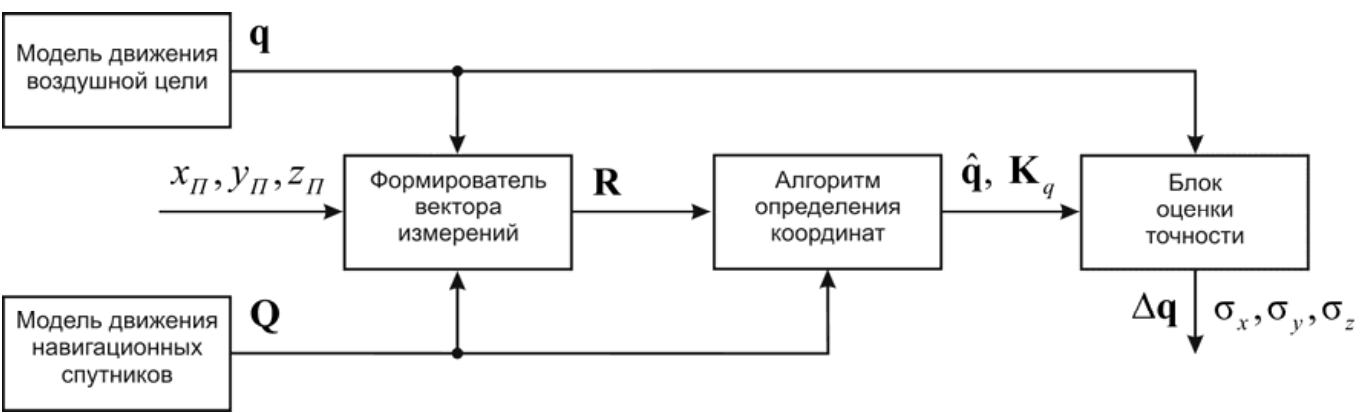

Рис. 2. Структурная схема имитационной модели 
стикам алгоритмов первичной обработки рассеянных навигационных сигналов в модернизированном приемнике ГНСС [9].

Алгоритм определения вектора координат воздушной цели на основе вектора измерений разностей дальностей $\mathbf{R}$ и матрицы координат навигационных спутников $\mathbf{Q}$ в соответствии с (7) - (14) формирует оценку $\hat{\mathbf{q}}$ вектора координат воздушной цели и ковариационной матрицы погрешностей $\mathbf{K}_{q}$.

Блок оценки точности определения координат предназначен для определения точности полученной оценки $\hat{\mathbf{q}}$ путем ее сравнения с истинным вектором состояния $\mathbf{q}$ и формирования вектора абсолютных ошибок оценки координат

$$
\Delta \mathbf{q}=\hat{\mathbf{q}}-\mathbf{q}
$$

Сравнение оценок вектора координат воздушной цели с истиной траекторией осуществляется отдельно по каждой координате и представляется в виде графиков временных зависимостей абсолютных ошибок $\Delta x=\hat{x}-x, \Delta y=\hat{y}-y, \Delta z=\hat{z}-z$. На этих же графиках отражается динамика изменения СКО формируемой оценки по соответствующим координатам $2 \sigma_{x}, 2 \sigma_{y}$, $2 \sigma_{z}$, где $\sigma_{x}^{2}, \sigma_{y}^{2}, \sigma_{z}^{2}$ - диагональные элементы формируемой ковариационной матрицы $\mathbf{K}_{q}$ погрешностей.

Кроме того, в этом же блоке формируется график временной зависимости абсолютной радиальной погрешности определения координат воздушной цели $\Delta R=\left[(\hat{x}-x)^{2}+(\hat{y}-y)^{2}+(\hat{z}-z)^{2}\right]^{1 / 2}$. На этом же графике отражается динамика изменения СКО формируемой радиальной погрешности $2 \sigma_{R}$, где $\sigma_{R}=\left[\sigma_{x}^{2}+\sigma_{y}^{2}+\sigma_{z}^{2}\right]^{1 / 2}$.

\section{Результаты эксперимента}

На рис. 3 показана геометрия проведенного численного эксперимента, где сплошной кривой обозначена истинная 3-D-траектория полета воздушной цели типа F-35. Полет воздушной цели осуществлялся в районе аэродрома вылета на удалении не более 5 км и на высоте не более 4 км.

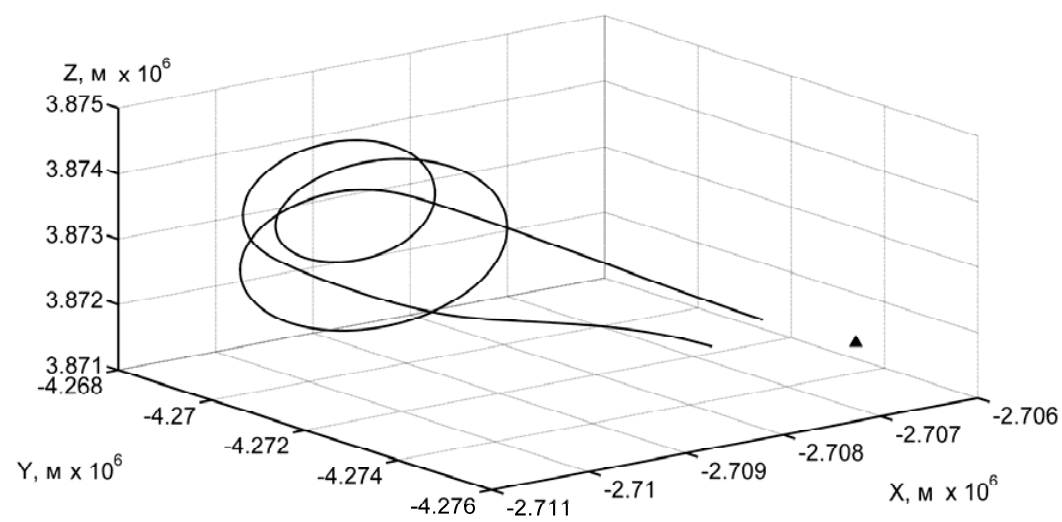

Рис. 3. Геометрия численного эксперимента 
На этом же рисунке жирным треугольником показано место установки наземного приемника многопозиционной системы наблюдения. Место положения приемника было выбрано условно, в удалении 1 км по оси $X$ и по оси $Y$ от точки взлета воздушной цели, из соображений попадания траектории ее полета в зону действия системы наблюдения. Координаты наземного приемника $\left(x_{\Pi}, y_{\Pi}, z_{\Pi}\right)$ использовались в качестве начального приближения для решения системы уравнений (6). Среднеквадратическое отклонение (СКО) случайной ошибки разностнодальномерных измерений было выбрано $\sigma_{\varepsilon}=5$ м и для простоты считалось постоянным на протяжении всего сеанса измерений, т.е. не зависело от ракурса наблюдения и высоты полета воздушной цели.

Результаты проведенного численного эксперимента показаны на рис. 4-7 в виде временных зависимостей значений элементов вектора абсолютных ошибок $\Delta \mathbf{q}$, формируемого на выходе блока оценки точности определения координат. График погрешности получаемой оценки $\Delta x$ приведен на рис. 4 (тонкая линия), где также показана динамика изменения СКО формируемой оценки по соответствующей координате $\pm 2 \sigma_{x}$ (жирная линия).

Аналогично графики погрешностей $\Delta y$ и $\Delta z$, а также СКО погрешности формируемых оценок показаны на рис. 5 и 6 соответственно.

Анализ рис. 4-6 позволяет сделать заключение о том, что точность формируемых оценок соответствует расчетному уровню и, в основном, не превышает удвоенного СКО, значение которого составило 10, 9 и 13 м соответственно по координатам $x, y, z$.

Временная зависимость абсолютной радиальной погрешности определения координат воздушной цели $\Delta R$ показана на рис. 7 (тонкая линия), жирной линией показана динамика изменения СКО формируемой оценки $2 \sigma_{R}$. На рис. 7 видно, что точность формируемой оценки соответствует расчетному уровню $\Delta R \leq 2 \sigma_{R}$ и, в основном, не превышает 18 м.

Анализ полученных в ходе имитационного моделирования результатов показывает, что при условии достаточной энергетики рассеянного сигнала и высокой точности дальномерных измерений в канале первичной обработки модернизированного приемника ГНСС использование предложенного способа в канале вторичной обработки обеспечивает точность определе-

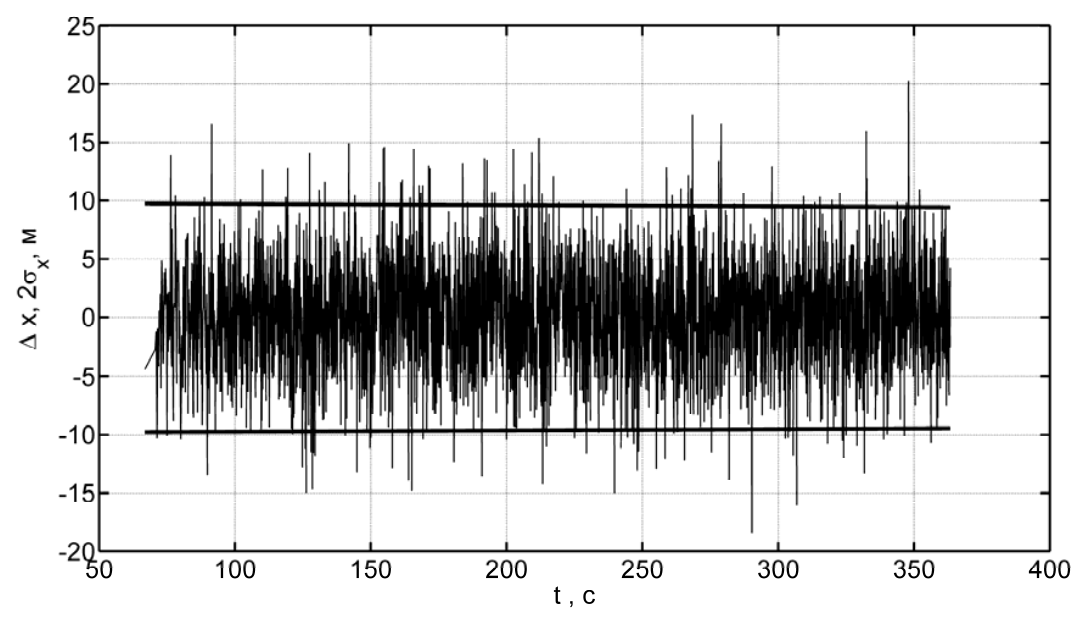

Рис. 4. Точность оценки координаты $x$ воздушной цели 


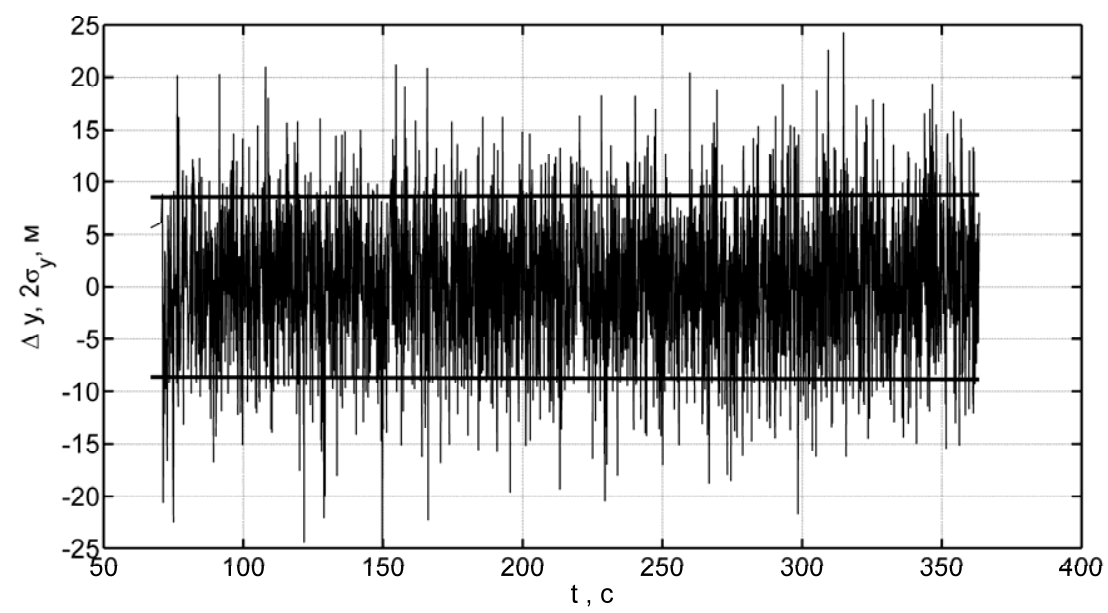

Рис. 5. Точность оценки координаты $y$ воздушной цели

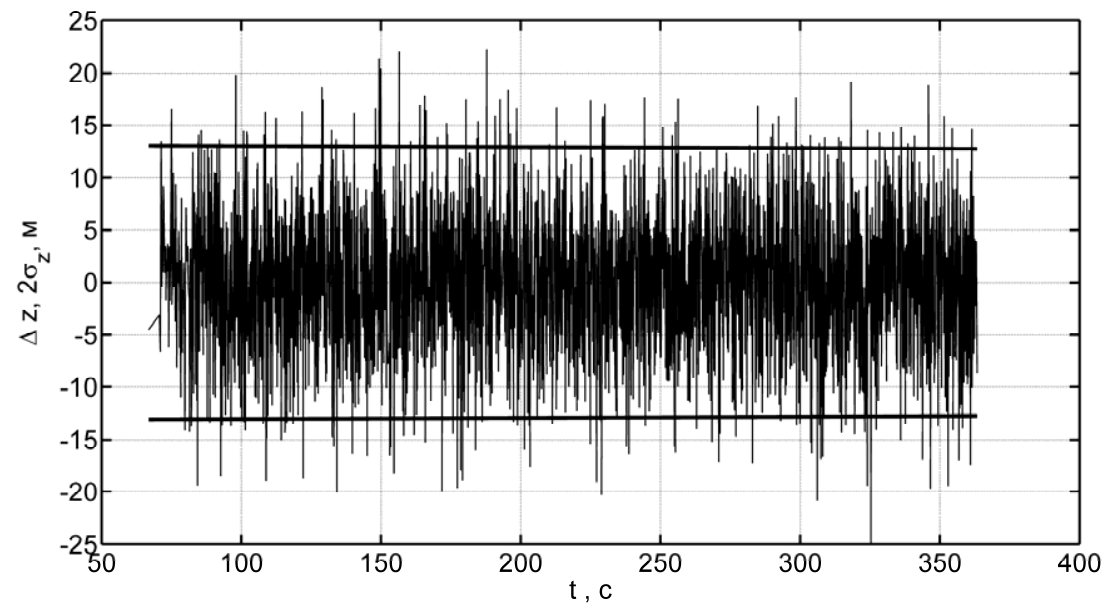

Рис. 6. Точность оценки координаты $z$ воздушной цели

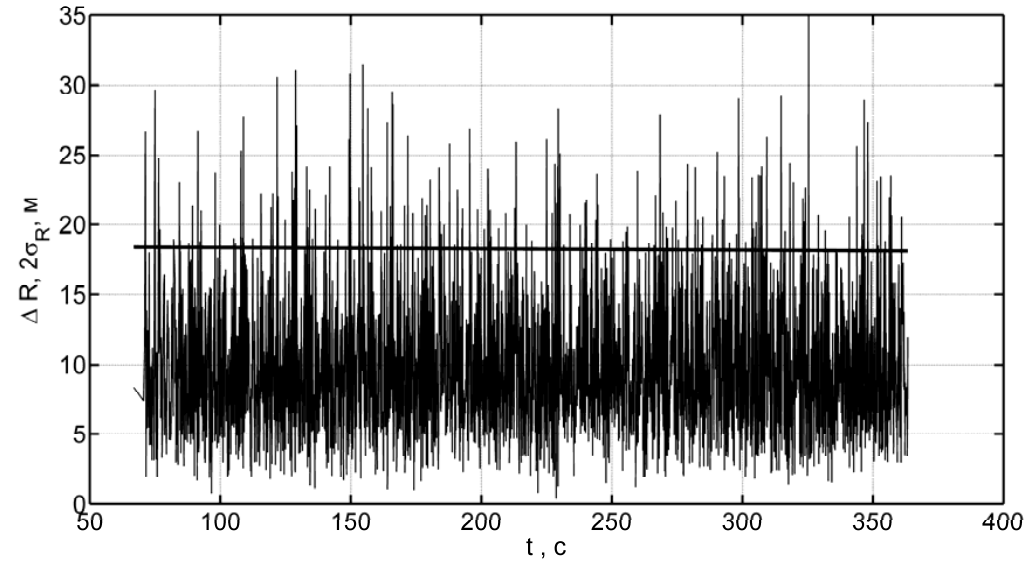

Рис. 7. Временная зависимость радиальной погрешности оценки местоположения воздушной цели 
ния координат воздушной цели, попавшей в зону действия многопозиционной системы наблюдения «навигационные спутники - воздушная цель - наземный приемник», соизмеримую с точностью оценки собственных координат приемника [12-15].

\section{Заключение}

Таким образом, в настоящей статье предложен новый способ определения координат воздушной цели в многопозиционной неизлучающей системе наблюдения «навигационные спутники - воздушная цель - наземный приемник», в основе которого лежит разностнодальномерный метод решения навигационной задачи в аппаратуре потребителя ГНСС. Проведенное с принятыми ограничениями численное моделирование показало, что предложенный способ позволяет с высокой точностью, присущей навигационным методам, определять в наземном приемнике координаты воздушной цели, попавшей в зону действия многопозиционной неизлучающей радиолокационной системы наблюдения «навигационные спутники - воздушная цель - наземный приемник», используя при этом информацию, содержащуюся в рассеянном воздушной целью навигационном сигнале ГНСС.

\section{Список литературы}

[1] Черняк В.С. Многопозииионная радиолокащия. М.: Радио и связь, 1993. 416 с. [Chernyak V.S. Multistation radiolocation. Moscow: Radio i sv'yaz, 1993. 416 p. (in Russian)]

[2] Фиолентов А.С. Новые технические средства разведки воздушных целей. Зарубежное военное обозрение, 2000, 4, 23-27 [Fiolentov A.S. New hardware components of survey of air targets. Foreign military review, 2000, 4, 23-27 (in Russian)]

[3] Griffiths H.D., From a different perspective: principles, practice and potential of bistatic radar, Proc. of the International Radar Conference, Adelaide, Australia, 2003, 1-7.

[4] Семашко П.Г., Пархоменко Н.Г., Охрименко А.Е. Потенциальные характеристики радиолокаторов с цифровым телевизионным подсветом. Успехи современной радиоэлектрони$\kappa u$, 2011, 10, 8-11 [Semashko P.G., Parhomenko N.G., Ohrimenko A.E. Potential characteristics of radiolocators with a digital television signal. Telecommunications and Radio Engineering, 2011, 10, 8-11 (in Russian)]

[5] Петров В.Н, Гришулин С.А. Наземные радиолокационные станции ПВО-ПРО на ТВД стран НАТО (Часть 2). Зарубежное военное обозрение, 2010, 9, 7-12 [Petrov V.N., Grishulin S.A. Land radar stations of air defence and antimissile defence on a battlefield of the countries of the NATO (the Part 2). Foreign military review, 2010, 9, 7-12 (in Russian)]

[6] Ксендзук А.В, Фатеев В.Ф., Попов С.А. Неизлучающая радиолокационная система, основанная на приёме отражённых сигналов навигационных систем ГЛОНАCС и GPS, Труды OAO «МАК «Вымпел», Сборник статей, М.: Радиотехника, 2009, 60-66 [Ksendzuk A.V., Fateev V.F., Popov S.A., Not radiating radar-tracking system based on reception of reflected signals of navigating systems GLONASS and GPS, Works of Open Society «MAK «Vympel», Moscow: Radioengineering, 2009, 60-66 (in Russian)]

[7] Беспалый В.Д. Перспективная многопозиционная радиолокационная система на основе сигналов спутниковых радионавигационных систем, Материалы II Всероссийской научной конференции с международным участием, Красноярск, 2007, 271-273 [Bespaliy V.D., The perspectiv 
multistation radar-tracking system on the basis of signals of satellite radio navigating systems, Materials of II All-Russia scientific conference with the international participation, Krasnoyarsk, 2007, 271-273 (in Russian)]

[8] Кирюшкин В.В., Черепанов Д.А. Бистатическая локация воздушных целей сигналами спутниковых радионавигационных систем. Вестник Воронежского государственного технического университета, 2010, 11(6), 33-38 [Kiryushkin V.V., Cherepanov D.A. Bistatic location of air targets by signals of satellite radio navigating systems. The bulletin of the Voronezh state technical university, 2010, 6(11), 33-38 (in Russian)]

[9] Кирюшкин В.В., Черепанов Д.А., Дисенов А.А., Ткаченко С.С. Способ обнаружения и оценки радионавигационных параметров сигнала космической системы навигации, рассеянного воздушной целью, и устройство его реализации. Патент РФ на изобретение №2014101847 (002722) от 21.01.2014 [Kiryushkin V.V., Cherepanov D.A., Disenov A.A., Tkachenko S.S. Method of detection and estimation of radio-navigation parameters of a space navigation system signal, dispersed by an air target, and the device of its implementation. The patent of the Russian Federation for the invention №2014101847 (002722), 21.01.2014 (in Russian)]

[10] Дмитриев П.П., Шабшеевич В.С. Сетевые спутниковые радионавигационные системы. М.: Радио и связь, 1982. 272 с. [Dmitriev P.P., Shabsheevich V.S. Network satellite radio navigating systems. Moscow: Radio i sv'yaz, 1982. 272 p. (in Russian)]

[11] Сосулин Ю.Г., Костров В.В., Паршин Ю.Н. Оценочно-корреляционная обработка сигналов и компенсация помех. М.: Радиотехника, 2014. 632 c. [Sosulin Yu.G., Kostrov V.V., Parshin Yu.N. The estimate-correlative signal processing and compensating of noises. Moscow: Radioengineering, 2014. 632 p. (in Russian)]

[12] Перов А.И., Харисов В.Н. ГЛОНАСС. Принщипь построения и функционирования. М.: Радиотехника, 2005. 688 c. [Perov A.I., Harisov V.N. GLONASS. Construction and functioning principles. Moscow: Radioengineering, 2005. 688 p. (in Russian)]

[13] Wang Jian-Guo. Test Statistics in Kalman Filtering. Journal of Global Positioning Systems, 2008, 7(1), 81-90.

[14] Абдалла Х.М., Кирюшкин В.В. Оценка вектора состояния высокодинамичных летательных аппаратов с использованием сигма-точечного фильтра Калмана с нелинейной моделью динамики объекта. Теория и техника радиосвязи, 2014, 2, 67-73 [Abdalla Kh.M., Kiryushkin V.V. Estimating state vector of high maneuvering aircrafts using the Unscented Kalman filter with nonlinear aircraft dynamics model. The theory and technics of a radio communication, 2014, 2, 67-73 (in Russian)]

[15] Гарматенко И.С. Международные стандарты оценки точности навигационной информации. Журнал Сибирского федерального университета. Техника и технологии. 2013, 6(8), 863-866 [Garmatenko I.S. The international standards of an estimation of accuracy of the navigating information. J. Sib. Fed. Univ. Eng. technol., 2013, 6(8), 863-866 (in Russian)] 